\title{
Automated generation and comparison of Takagi-Sugeno and polytopic quasi-LPV models
}

\author{
Damiano Rotondo ${ }^{\mathrm{a}, *}$, Vicenç Puiga, ${ }^{\mathrm{a},}$ Fatiha Nejjari ${ }^{\mathrm{a}}$, Marcin Witczak ${ }^{\mathrm{c}}$ \\ ${ }^{a}$ Advanced Control Systems (SAC), Universitat Politècnica de Catalunya (UPC), Edifici TR11, Rambla Sant \\ Nebridi 10, 08222 Terrassa, Spain. \\ ${ }^{b}$ Institut de Robotica i Informatica Industrial (IRI), UPC-CSIC, Carrer de Llorens i Artigas, 4-6, 08028 \\ Barcelona, Spain. \\ ${ }^{c}$ Institute of Control and Computation Engineering, University of Zielona Góra, ul. Podgórna 50, 65-246 \\ Zielona Góra, Poland.
}

\begin{abstract}
In the last decades, gain-scheduling control techniques have consolidated as an efficient answer to analysis and synthesis problems for non-linear systems. Among the approaches proposed in the literature, the linear parameter varying (LPV) and TakagiSugeno (TS) paradigms have proved to be successful in dealing with the different trials that the analyzer, or the designer, of a gain-scheduled control system has to face. Despite the strong similarities between the two paradigms, research on LPV and TS systems has been performed in an independent way and some results that could be useful for both paradigms were obtained only for one of them. However, in recent works, some clues that there is a very close connection between LPV and TS worlds have been presented. The present paper openly addresses the presence of strong analogies between LPV and TS models, in an attempt to establish a bridge between these two worlds, so far considered different. In particular, this paper addresses the modeling problem, presenting two methods for the automated generation of LPV and TS systems introducing some measures in order to compare the obtained models. A mathematical example is used to illustrate the proposed methods.
\end{abstract}

Keywords: Modeling, Takagi-Sugeno (TS) fuzzy systems, Linear parameter varying (LPV) systems.

\section{Introduction}

In the last decades, gain-scheduling control techniques have consolidated as an efficient answer to analysis and synthesis problems for non-linear systems [1]. The strength of these techniques consists in the fact that the properties of the non-linear

\footnotetext{
${ }^{*}$ Corresponding author

Email addresses: damiano.rotondo@yahoo.it (Damiano Rotondo), vicenc.puig@upc.edu (Vicenç Puig), fatiha.nejjari@upc. edu (Fatiha Nejjari), m.witczak@issi.uz.zgora.pl (Marcin Witczak)
} 
systems are expressed by a collection of linear subsystems, which are also used for designing the controller. This is realized in a divide and conquer fashion so that well established linear methods can be applied to non-linear problems. Two approaches, among others, have proved to be successful in dealing with the different trials that the analyzer, or the designer, of a gain-scheduled control system has to face: the linear parameter varying (LPV) and the Takagi-Sugeno (TS) paradigms.

LPV systems were introduced by Shamma [2] to distinguish such systems from linear time invariant (LTI) and linear time varying (LTV) ones [3]. Since then, the LPV paradigm has become a standard formalism in systems and control, for analysis, controller synthesis and even system identification. This class of systems is important because gain-scheduling control of non-linear systems can be performed according to the LPV paradigm, where the non-linearity is embedded in the varying parameters that depend on some endogenous signals, e.g. some system states (in this case, the system is referred to as quasi- $L P V$, to make a further distinction with respect to pure LPV systems, where the varying parameters only depend on exogenous signals). Among the practical applications where the LPV paradigm has been successfully applied, there are: missiles [4], aircrafts [5, 6], energy production systems [7, 8], robotic systems [9], active suspension of vehicles [10], engines [11] and fault tolerant control [12].

On the other hand, Takagi-Sugeno systems, introduced in [13], basically provide an effective way of representing non-linear systems with the aid of fuzzy sets, fuzzy rules and a set of local linear models. The overall model of the system is obtained by merging the local models through fuzzy membership functions. The TS paradigm has been successfully applied in the same fields where the LPV one proved to be successful: missiles [14], aircrafts [15], energy production systems [16], robotic systems [17], active suspension of vehicles [18], engines [19] and fault tolerant control [20].

Despite the strong similarities of the two paradigms, LPV and TS systems have nearly always been treated as though as they belonged to two different worlds. In fact, the research for each of them has been performed in an independent way and such that cross-references between papers dealing with the LPV theory and those dealing with the TS theory are quite uncommon. As a consequence, some theoretical results that could be useful for both types of systems have been applied only to one type, waiting for the researchers to discover that they could be applied to the other type as well.

However, in some recent works, some clues that there is a close connection between the LPV and the fuzzy TS paradigms have been presented [21, 22]. In [23], Rong and Irwin have pointed out that LPV systems can describe Takagi-Sugeno fuzzy models if the "scheduling functions" of the former paradigm are treated as membership functions of the latter one. Bergsten and his co-workers [24] point out that, since it has been proved that a TS fuzzy system, where the local affine dynamic models are offequilibrium local linearizations, leads to an arbitrarily close approximation of an LTV dynamical system about an arbitrary trajectory [25], the results concerning observers for TS fuzzy systems are also relevant to LPV systems. In [26], Collins has commented that, even though the results in [27] seem to be very related to existing results on LPV control, they are not put in perspective with those existing for LPV systems. He also claimed that it is apparent that the fuzzy T-S model is a special case of an LPV model. However, even if from theoretical analysis and design points of view it is difficult to find clear differences between the two paradigms [28], LPV and TS systems are still 
considered different and their equality is dubious [29].

The present paper openly addresses the presence of strong analogies between LPV and TS models, in an attempt to establish a bridge between these two worlds, so far considered to be different. In particular, this paper considers the modeling problem, with the following important contributions:

- The analogies and connections between LPV and TS systems are clearly stated;

- it is shown that the method for the automated generation of LPV models by non-linear embedding presented in [30] can be easily extended to solve the corresponding problem for TS models;

- it is shown that the method for the generation of a TS model for a given nonlinear multivariable function based on the sector non-linearity concept [31], can be extended to the problem of generating a polytopic LPV model for a given non-linear dynamical system;

- two measures are proposed in order to compare the obtained models and choose which one can be considered the best one. The first measure is based on the notion of overboundedness. The second measure is based on region of attraction estimates and quadratic $\mathcal{D}$-stabilizability in linear matrix inequality (LMI) regions;

- using a mathematical example, an application of the proposed methodologies is performed;

Notice that the resulting method for automated generation of TS models by nonlinear embedding has been already used by the fuzzy community in an intuitive way. For example, one can verify that the TS models obtained by Tanaka and Wang in [27], are contained within the set of TS models obtained through the method proposed in this paper. In the present work, the method used in [27] is automated adapting a technique developed by the LPV community that had never been used for TS systems until now.

The paper has the following structure: in Section 2, LPV and TS systems are presented and their analogies are highlighted. In Section 3, two measures to compare models are introduced. In Section 4, the method for automated generation of TS models by non-linear embedding is presented. The same is done in Section 5, for the automated generation of polytopic LPV models via sector non-linearity concept. Section 6 presents the mathematical example for the application of the two techniques. Finally, in Section 7, some conclusions are outlined.

\section{LPV and TS systems definitions}

\subsection{LPV systems}

Following the notation used by [32], $\sigma$ stands for the Laplace variable $s$ in the continuous-time case and for the Z-transform variable $z$ in the discrete-time case. Similarly, $\tau$ will stand for the time $t \in \mathbb{R}^{+}$in the continuous-time case and $k \in \mathbb{Z}^{+}$for the time samples in the discrete-time case. The notation $\sigma \cdot x(\tau)$ stands for $\dot{x}(t)$ for continuous-time systems and for $x(k+1)$ for discrete-time systems. 
Then, an LPV system is defined as a linear system whose coefficients depend on some varying parameter $\theta(\tau) \in \mathbb{R}^{n_{\theta}}$, assumed to be unknown a priori, but measured or estimated in real-time [33]:

$$
\begin{gathered}
\sigma . x(\tau)=A(\theta(\tau)) x(\tau)+B(\theta(\tau)) u(\tau) \\
y(\tau)=C(\theta(\tau)) x(\tau)+D(\theta(\tau)) u(\tau)
\end{gathered}
$$

where $x \in \mathbb{R}^{n_{x}}, u \in \mathbb{R}^{n_{u}}$ and $y \in \mathbb{R}^{n_{y}}$ are the state, the input and the output vector, respectively, and $A, B, C$ and $D$ are varying matrices of appropriate dimensions.

An LPV system is called polytopic when it can be represented by state-space matrices $A(\theta(\tau)), B(\theta(\tau)), C(\theta(\tau))$ and $D(\theta(\tau))$, where the parameter vector $\theta(\tau)$ ranges over a fixed polytope $\Theta$, and the dependence of $A(\theta(\tau)), B(\theta(\tau)), C(\theta(\tau))$ and $D(\theta(\tau))$ on $\theta$ is affine [32], resulting in the following:

$$
\begin{gathered}
\sigma . x(\tau)=\sum_{i=1}^{N} \pi_{i}(\theta(\tau))\left(A_{i} x(\tau)+B_{i} u(\tau)\right) \\
y(\tau)=\sum_{i=1}^{N} \pi_{i}(\theta(\tau))\left(C_{i} x(\tau)+D_{i} u(\tau)\right)
\end{gathered}
$$

where the quadruple $\left(A_{i}, B_{i}, C_{i}, D_{i}\right)$ defines the so-called vertex system and $\pi_{i}$ are the non-negative coefficients of the polytopic decomposition such that:

$$
\sum_{i=1}^{N} \pi_{i}(\theta(\tau))=1 \quad, \quad \pi_{i}(\theta(\tau)) \geq 0 \quad \forall i=1, \ldots, N, \quad \forall \theta \in \Theta
$$

\subsection{TS systems}

TS systems, as proposed by Takagi and Sugeno [13], are described by local models merged together using fuzzy IF-THEN rules [27], as follows:

$$
\begin{aligned}
& \text { IF } \vartheta_{1}(\tau) \text { is } M_{i 1} A N D \ldots A N D \vartheta_{p}(\tau) \text { is } M_{i p} \\
& \quad T H E N\left\{\begin{array}{l}
\sigma \cdot x_{i}(\tau)=A_{i} x(\tau)+B_{i} u(\tau) \\
y_{i}(\tau)=C_{i} x(\tau)+D_{i} u(\tau)
\end{array} \quad i=1, \ldots, N\right.
\end{aligned}
$$

where $\vartheta_{1}(\tau), \ldots, \vartheta_{p}(\tau)$ are premise variables that can be functions of the state variables, external disturbances and/or time. Each linear consequent equation represented by $A_{i} x(\tau)+B_{i} u(\tau)$ is called a subsystem.

Given a pair $(x(\tau), u(\tau))$, the state and output of the TS system can easily be inferred:

$$
\begin{aligned}
\sigma \cdot x(\tau) & =\sum_{i=1}^{N} w_{i}(\vartheta(\tau))\left(A_{i} x(\tau)+B_{i} u(\tau)\right) \mid \sum_{i=1}^{N} w_{i}(\vartheta(\tau)) \\
= & \sum_{i=1}^{N} \rho_{i}(\vartheta(\tau))\left(A_{i} x(\tau)+B_{i} u(\tau)\right)
\end{aligned}
$$




$$
\begin{aligned}
y(\tau) & =\sum_{i=1}^{N} w_{i}(\vartheta(\tau))\left(C_{i} x(\tau)+D_{i} u(\tau)\right) / \sum_{i=1}^{N} w_{i}(\vartheta(\tau)) \\
& =\sum_{i=1}^{N} \rho_{i}(\vartheta(\tau))\left(C_{i} x(\tau)+D_{i} u(\tau)\right)
\end{aligned}
$$

where $\vartheta(\tau)=\left[\vartheta_{1}(\tau), \ldots, \vartheta_{p}(\tau)\right]$ is the vector containing the premise variables, and $w_{i}(\vartheta(\tau))$ and $\rho_{i}(\vartheta(\tau))$ are defined as follows:

$$
\begin{gathered}
w_{i}(\vartheta(\tau))=\prod_{j=1}^{p} M_{i j}\left(\vartheta_{j}(\tau)\right) \\
\rho_{i}(\vartheta(\tau))=\frac{w_{i}(\vartheta(\tau))}{\sum_{i=1}^{N} w_{i}(\vartheta(\tau))}
\end{gathered}
$$

where $M_{i j}\left(\vartheta_{j}(\tau)\right)$ is the grade of membership of $\vartheta_{j}(\tau)$ in $M_{i j}$ and $\rho_{i}(\vartheta(\tau))$ is such that:

$$
\left\{\begin{array}{l}
\sum_{i=1}^{N} \rho_{i}(\vartheta(\tau))=1 \\
\rho_{i}(\vartheta(\tau)) \geq 0, \quad i=1, \ldots, N
\end{array}\right.
$$

\subsection{Analogies between polytopic LPV and TS systems}

There are strong analogies between polytopic LPV and TS systems. In fact, the only remarkable difference between the two frameworks is the set of mathematical tools that are used for obtaining the system description. In the LPV case, these tools belong to the standard mathematics; on the other hand, in the TS case, they belong to the fuzzy theory. In particular, the correspondences between polytopic LPV and TS systems are between:

- the scheduling parameters $\theta$ of LPV systems and the premise variable $\vartheta$ of TS systems;

- the coefficients of the polytopic decomposition $\pi_{i}$ and the coefficients $\rho_{i}$ that describe the level of activation of each local model;

- the vertex systems in the polytopic LPV case and the subsystems in the TS case.

These analogies can be strongly exploited for extending techniques and results that have been developed or found for polytopic LPV systems to the TS case, and viceversa.

\section{Measures for comparison between LPV and TS models}

\subsection{Overboundedness-based measure}

Given a non-linear system:

$$
\sigma \cdot x=f(x, u, w)
$$




$$
y=g(x, u, w)
$$

where $w \in \mathbb{R}^{n_{w}}$ is some exogenous signal and $y \in \mathbb{R}^{n_{y}}$ is the output, the approaches for automated generation of polytopic LPV and TS models proposed in this paper (see Sections 4 and 5) provide a systematic methodology for building a whole set of LPV/TS models representing the non-linear system (12)-(13). Hence, it is interesting to compare the obtained models in order to choose which one is the best. This is especially important from the point of view of the fault tolerant control for nonlinear systems [34], for which the model quality is of paramount importance.

Hereafter, a measure based on the notion of overboundedness is proposed, similar to the one proposed in [30]. The idea is to calculate the volume of the (hyper)region contained between the vertices/subsystems (hyper)planes: the smaller is this volume, the better is the approximation offered by the polytopic LPV/TS model. To obtain the measure, subsets $S_{1}, \ldots, S_{n_{x}}$ of $\left\{\mathbb{X}, \mathbb{U}, \mathbb{W}, \mathbb{F}_{1}\right\}, \ldots,\left\{\mathbb{X}, \mathbb{U}, \mathbb{W}, \mathbb{F}_{n_{x}}\right\}$ must be chosen, where $\mathbb{X}, \mathbb{U}, \mathbb{W}$ and $\mathbb{F}_{i}$ are the state space, the input space, the exogenous signal space and the $i$-th state variable derivative space, respectively. Then, if $V_{1}^{(S)}, \ldots, V_{n_{x}}^{(S)}$ are the volumes of the subsets $S_{1}, \ldots, S_{n_{x}}$, and $V_{1}, \ldots, V_{n_{x}}$ are the volumes of the (hyper)regions contained between the vertices/subsystems (hyper)planes in $S_{1}, \ldots, S_{n_{x}}$, a measure of the goodness of the polytopic LPV/TS model is given by:

$$
M=\frac{V_{1} V_{2} \cdots V_{n_{x}}}{V_{1}^{(S)} V_{2}^{(S)} \cdots V_{n_{x}}^{(S)}}
$$

where the smaller is this measure, the better is the model ${ }^{1}$.

Notice that in some situations, calculating the volumes $V_{1}, \ldots, V_{n_{x}}$ can be a hard task. Then, an approximate measure can be used as follows:

$$
\tilde{M}=\frac{\tilde{V}_{1} \tilde{V}_{2} \cdots \tilde{V}_{n_{x}}}{V_{1}^{(S)} V_{2}^{(S)} \cdots V_{n_{x}}^{(S)}}
$$

where $\tilde{V}_{i}$ is an approximation of $V_{i}$. In particular, in this paper, each factor $\tilde{V}_{i} / V_{i}^{(S)}$ is obtained generating randomly a certain number $N$ of points inside the subset $S_{i}$, and then calculating the ratio between the points that can be described by a polytopic combination through the model taken into consideration, and the total number of points. Obviously, $\tilde{M}$ approaches $M$ in the limit as $N \rightarrow \infty$. However, it is impossible to set $N=\infty$. Thus, the problem becomes the one of selecting $N$ in such a way that $\tilde{M}$, i.e. the estimation of $M$, has some desired properties. In order to do this, notice that the process of generating points in the subset $S_{i}$ and checking whether or not they can be described by the model taken into consideration is a Bernoulli process [35] with a limited number $N$ of Bernoulli trials. Hence, the estimator $\tilde{M}$ can be analyzed using

\footnotetext{
${ }^{1}$ The measure $M$ usually decreases when the number of vertex systems/subsystems used in the considered polytopic LPV/TS model increases. In some cases, e.g. controller synthesis, this could lead to an increase in the computational effort that is not taken into account by the proposed measure $M$. If it is desired to include such an effect in the evaluation of the goodness of the model, a slight modification of $M$ should be done.
} 
the results coming from the theory of statistics and probability [36].

\subsection{Region of attraction estimates-based measure}

Stability analysis and controller synthesis for LPV and TS systems have been an important topic of research in the last decades. One of the most used tools to deal with the problem of analyzing the stability or designing a stabilizing controller for these systems is the quadratic stabilizability condition [37], thanks to which a common Lyapunov matrix can be obtained such that the controller stabilizes the closed-loop system using LMIs. This condition is appealing due to its numerical simplicity, and has often been preferred to other conditions involving more complex Lyapunov functions, e.g. parameter-dependent [38].

It is often believed that a closed loop quasi-LPV/TS system, obtained from a nonlinear system using an exact transformation procedure as the one presented in this paper, that satisfies stability (or some other goal) for all parameters varying in a convex region, e.g. a bounding box, implies that stability is satisfied for the underlying nonlinear system. This is not always true, as shown in [39], where a Van der Pol equation with reversed vector field example was used to demonstrate that the LPV/TS analysis of the nonlinear system does not guarantee local asymptotic stability. However, [39] also shows that the LPV/TS analysis can be used to estimate the region of attraction for the underlying nonlinear system. In fact, even though finding the exact region of attraction analytically might be difficult or even impossible [40], the Lyapunov functions can be used to estimate the region of attraction.

Assume that:

$$
\sigma \cdot x(\tau)=A(\theta(x(\tau))) x(\tau)
$$

satisfies some stability and performance conditions [41,37,42] for $\theta \in \Theta$ in the sense of decreasing the Lyapunov function $V(x(\tau))=x(\tau)^{T} X x(\tau)$ with $X>0$.

Moreover, let us define the following sets:

$$
\begin{gathered}
\mathcal{X}=\{x \in D \mid \theta(x) \in \Theta\} \\
\Gamma_{\beta}=\{x \in D \mid V(x) \leq \beta\}
\end{gathered}
$$

and, for the nonlinear system:

$$
\sigma \cdot x(\tau)=f(x(\tau))
$$

with the origin as an equilibrium point, let us define the region of attraction as the set:

$$
R_{A}=\left\{x(0) \mid \lim _{\tau \rightarrow \infty} \phi(\tau ; x(0))=0\right\}
$$

where $\phi(\tau ; x(0))$ denotes the solution that starts at initial state $x(0)$ at time $t=0$.

Then, the following theorem holds:

Theorem 1. Consider the nonlinear system (19), with the exact quasi-LPV representation (16). If $\Gamma_{\beta} \subseteq \mathcal{X}$ then $\Gamma_{\beta} \subseteq R_{A}$, where $R_{A}$ is the region of attraction.

Proof: See [39]. 
A consequence of this theorem is that a region of attraction approximation is given by the (hyper)ellipsoid provided by the positive definite matrix $X$ of the Lyapunov function. In this paper, a measure based on the region of attraction approximation is proposed in order to compare quasi-LPV and TS models obtained from the same nonlinear system. This measure is defined as follows:

$$
M_{\beta}=\frac{V_{\beta}}{V_{\Theta}}
$$

where $V_{\beta}$ is the volume of $\Gamma_{\beta}$, and $V_{\Theta}$ is the volume of the polytopic region $\mathcal{X}$ within which the parameter vector $\theta$ (or the premise variables $\vartheta$ in the case of TS representation) can take values.

Additionally, the LMI pole placement conditions introduced by [42] will be used to define the goal to be achieved by the control system. It should be pointed out that other criteria can be introduced and easily incorporated within the general framework provided in the subsequent part of this paper, e.g. $\mathcal{H}_{\infty}$ norm [43].

A subset $\mathcal{D}$ of the complex plane is called an LMI region if there exist a symmetric matrix $\alpha=\left[\alpha_{k l}\right]_{1 \leq k, l \leq m} \in \mathbb{R}^{m \times m}$ and a matrix $\beta=\left[\beta_{k l}\right]_{1 \leq k, l \leq m} \in \mathbb{R}^{m \times m}$ such that:

$$
\mathcal{D}=\left\{z \in \mathbb{C}: f_{\mathcal{D}}(z)<0\right\}
$$

with:

$$
f_{\mathcal{D}}(z)=\alpha+z \beta+\bar{z} \beta^{T}=\left[\alpha_{k l}+\beta_{k l} z+\beta_{l k} \bar{z}\right]_{1 \leq k, l \leq m}
$$

Then, an LTI system is said to be $\mathcal{D}$-stable if it is stable and if all its poles lie in $\mathcal{D}$ $[42,44]$.

Following [45] and with a little abuse of language, the poles of an LPV system are defined as the set of all the poles of the LTI systems obtained by freezing $\theta$ to all its possible values in $\Theta$. In [46], the quadratic $\mathcal{D}$-stability of LPV systems was defined as follows (a similar definition holds in case of TS systems):

Definition 1. An LPV system $\sigma \cdot x(\tau)=A(\theta(\tau)) x(\tau)$ is quadratically $\mathcal{D}$-stable, with $\mathcal{D}$ an LMI region defined as in (22)-(23), if there exists a symmetric positive definite matrix $X>0$ such that $\forall \theta \in \Theta$ :

$$
\left\{\begin{array}{l}
A(\theta) X+X A^{T}(\theta)<0 \quad \text { if } \sigma=t \\
\left(\begin{array}{cc}
-X \quad A(\theta) X \\
X A^{T}(\theta)-X
\end{array}\right)<0 \quad \text { if } \sigma=k \\
{\left[\alpha_{k l} X+\beta_{k l} A(\theta) X+\beta_{l k} X A^{T}(\theta)\right]_{1 \leq k, l \leq m}<0}
\end{array}\right.
$$

Hence, given the polytopic LPV system (3) (or the TS system (6)), it is possible to design a Parallel Distributed Compensation (PDC) controller [47], which is a very popular approach for both LPV/TS systems:

$$
u(\tau)=K(\theta(\tau)) x(\tau)=\sum_{i=1}^{N} \pi_{i}(\theta(\tau)) K_{i} x(\tau)
$$


or a TS controller:

$$
\begin{gathered}
I F \vartheta_{1}(\tau) \text { is } M_{i 1} A N D \ldots A N D \vartheta_{p}(\tau) \text { is } M_{i p} \\
T H E N u(\tau)=K_{i} x(\tau) \quad i=1, \ldots, N
\end{gathered}
$$

using the following theorem:

Theorem 2. Let $\mathcal{D}$ be an LMI region with characteristic function (23), and assume that there exists a single Lyapunov matrix $X>0$ and $N$ matrices $\Gamma_{i}$ such that the following set of LMIs is feasible $(i=1, \ldots, N, j=1, \ldots, N)$ :

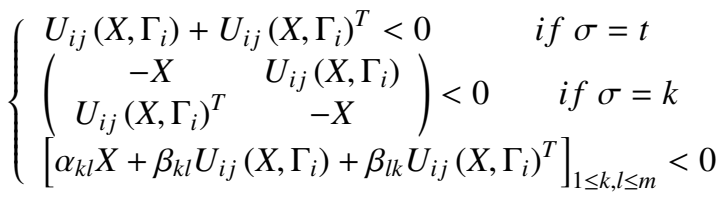

with:

$$
U_{i j}\left(X, \Gamma_{i}\right)=A_{i} X+B_{j} \Gamma_{i}
$$

Then, the LPV system (3) (or the TS system (6)) with the state-feedback controller (25) (or (26)), whose vertex gains are calculated as $K_{i}=\Gamma_{i} X^{-1}$, is quadratically $\mathcal{D}$ stable.

Proof: The proof is similar to the one of the Theorem presented in [42], and makes extensive use of the basic property of matrices [48] that any linear combination of positive (negative) definite matrices with non-negative coefficients, whose sum is positive, is positive (negative) definite.

\section{Generation of TS models via non-linear embedding}

A method for the automated generation of LPV models, when affine or polytopic models are desired, has been presented in [30]. These models are generated from a general non-linear model by hiding the non-linearities in the scheduling parameters. In this section, it is shown that this method can be used for generating a Takagi-Sugeno model from a given non-linear model.

Consider the non-linear state ${ }^{2}$ equation (12). The automated generation of TS models via non-linear embedding consists of the following five steps:

- In the first step, (12) is rewritten in a standard form, that is, each of its rows is expanded into its summands $f_{i j}$ :

$$
\sigma \cdot x_{i}=\sum_{j=1}^{T_{i}} f_{i j}(x, u, w), \quad i=1, \ldots, n_{x}
$$

\footnotetext{
${ }^{2}$ The method can be applied to the output equation (13) without significant differences.
} 
where $T_{i}$ is the total number of summands of that row. Then, each summand is decomposed into its numerator $\alpha_{i j}$, denominator $\beta_{i j}$ and constant factor $\kappa_{i j}$ :

$$
\sigma . x_{i}=\sum_{j=1}^{T_{i}} \kappa_{i j} \frac{\alpha_{i j}(x, u, w)}{\beta_{i j}(x, u, w)}, \quad i=1, \ldots, n_{x}
$$

Finally, the numerator is factored as the product of non-factorisable terms $h_{i j}$ and integer powers of the states $x_{q}, q=1, \ldots, n_{x}$ and the inputs $u_{r}, r=1, \ldots, n_{u}$ :

$$
\alpha_{i j}=\prod_{q=1}^{n_{x}} \prod_{r=1}^{n_{u}} h_{i j}(x, u, w) x_{q}^{\mu_{i j q}} u_{r}^{v_{i j r}}
$$

- In the second step, two classes of summands are distinguished: (a) constant or non-factorisable numerator, $\mathcal{K}_{0}$, when neither a power of the state $x_{i}$ nor of an input $u_{i}$ is a factor of the numerator; and (b) arbitrary positive power of factor, $\mathcal{K}_{P}$, when the summand has a numerator with positive integer powers of a state variable $x_{i}$ or input $u_{i}$;

- In the third step, according to the classification of each summand, components $\vartheta_{i j k}^{a}$ and $\vartheta_{i j k}^{b}$ that link the summand to the entries of the state and input matrices $A$ and $B$ are chosen. If the summand $f_{i j}$ belongs to $\mathcal{K}_{0}$, one can obtain $n_{x}$ possible assignments to the state matrix $A$ and $n_{u}$ possible assignments to the input matrix $B$, with $\vartheta_{i j k}^{a}$ and $\vartheta_{i j k}^{b}$ defined as follows:

$$
\begin{aligned}
& \vartheta_{i j k}^{a}=\kappa_{i j} \frac{\alpha_{i j}(x, u, w)}{\beta_{i j}(x, u, w) x_{k}}, \quad k=1, \ldots, n_{x} \\
& \vartheta_{i j k}^{b}=\kappa_{i j} \frac{\alpha_{i j}(x, u, w)}{\beta_{i j}(x, u, w) u_{k}}, \quad k=1, \ldots, n_{u}
\end{aligned}
$$

Otherwise, if the summand $f_{i j}$ belongs to $\mathcal{K}_{p}$, one can choose to assign the summand to an element of the state or input matrix, as long as the element is a factor of the numerator, i.e. if there exists a $k$ for which $\mu_{i j k} \neq 0$ or $v_{i j k} \neq 0$;

- In the fourth step, the premise variables $\vartheta$ are derived from $\vartheta_{i j k}^{a}$ and $\vartheta_{i j k}^{b}$. This can be done either by direct assignment or by superposition. In the direct assignment case, the premise variables are directly chosen as $\vartheta_{i j k}^{a}$ and $\vartheta_{i j k}^{b}$, such that:

$$
a_{i k}=\sum_{j=1}^{\zeta_{a}} \vartheta_{i j k}^{a} \quad b_{i k}=\sum_{j=1}^{\zeta_{b}} \vartheta_{i j k}^{b}
$$

where $\zeta_{a}$ and $\zeta_{b}$ are the number of components of the same equation $\sigma \cdot x_{i}$ that are assigned to the same state $x_{k}$ or input $u_{k}$, respectively, but have been obtained from different summands. In the superposition case, the premise variables, denoted by $\vartheta_{i k}^{a}$ and $\vartheta_{i k}^{b}$, are obtained through a sum of all the contributions of a 
summand to the same element of $A$ or $B$ :

$$
\vartheta_{i k}^{a}=\sum_{j=1}^{\zeta_{a}} \vartheta_{i j k}^{a} \quad \vartheta_{i k}^{b}=\sum_{j=1}^{\zeta_{b}} \vartheta_{i j k}^{b}
$$

such that the premise variables correspond to the elements of the state space matrices:

$$
a_{i k}=\vartheta_{i k}^{a} \quad b_{i k}=\vartheta_{i k}^{b}
$$

In both cases, the premise variables need to be renumbered in order to be coherent with the numbering presented in (6).

- In the final step, an adaptation of the technique used in [49] for obtaining polytopic LPV models, often referred to as bounding box method, is used to complete the generation of the TS model. The minimum and maximum values of each premise variable $\vartheta_{i}$ over the possible values of $x, u$ and $w$, are obtained as follows:

$$
\underline{\vartheta}_{i}=\min _{x, u, w} \vartheta_{i} \quad \bar{\vartheta}_{i}=\max _{x, u, w} \vartheta_{i}
$$

From the maximum and minimum values, $\vartheta_{i}$ can be represented as:

$$
\vartheta_{i}=M_{1 i}\left(\vartheta_{i}\right) \underline{\vartheta}_{i}+M_{2 i}\left(\vartheta_{i}\right) \bar{\vartheta}_{i}
$$

with the additional constraint:

$$
M_{1 i}\left(\vartheta_{i}\right)+M_{2 i}\left(\vartheta_{i}\right)=1
$$

such that the membership functions are calculated as:

$$
M_{1 i}\left(\vartheta_{i}\right)=\frac{\bar{\vartheta}_{i}-\vartheta_{i}}{\bar{\vartheta}_{i}-\underline{\vartheta}_{i}} \quad \text { and } \quad M_{2 i}\left(\vartheta_{i}\right)=\frac{\vartheta_{i}-\underline{\vartheta}_{i}}{\bar{\vartheta}_{i}-\underline{\vartheta}_{i}}
$$

Finally, the subsystems are obtained by considering each possible combination of membership functions in the IF clauses of the TS model.

An example of the application of the proposed technique is given in Section 6.1.

\section{Generation of polytopic LPV models via sector non-linearity concept}

The idea of using sector non-linearity in TS model construction first appeared in [50], where the single variable system case was considered, and extended to the multivariable case in [31]. In this section, it is shown that this method can also be used for generating a polytopic LPV model from a given non-linear model.

Consider the non-linear state equation (12), under the hypothesis that the function $f(x, u, w)$ is differentiable everywhere (as in the previous method, the application to the output equation (13) can be performed without significant differences). The automated 
generation of polytopic LPV models via sector non-linearity concept consists of the following steps:

- In the first step, the space $\{\mathbb{X}, \mathbb{U}, \mathbb{W}\}$ is partitioned into its $2^{n_{x}+n_{u}+n_{W}}$ quadrants. Each quadrant is denoted by:

$$
R\left(s_{1}^{(x)}, \ldots, s_{n_{x}}^{(x)}, s_{1}^{(u)}, \ldots, s_{n_{u}}^{(u)}, s_{1}^{(w)}, \ldots, s_{n_{w}}^{(w)}\right)
$$

where:

$$
\begin{gathered}
\left\{\begin{array}{c}
s_{j}^{(x)}=1 \Leftrightarrow x_{j} \geq 0 \\
s_{j}^{(x)}=0 \Leftrightarrow x_{j} \leq 0
\end{array}\right. \\
\left\{\begin{array}{c}
s_{j}^{(u)}=1 \Leftrightarrow u_{j} \geq 0 \\
s_{j}^{(u)}=0 \Leftrightarrow u_{j} \leq 0
\end{array}\right. \\
\left\{\begin{array}{c}
s_{j}^{(w)}=1 \Leftrightarrow w_{j} \geq 0 \\
s_{j}^{(w)}=0 \Leftrightarrow w_{j} \leq 0
\end{array}\right.
\end{gathered}
$$

Then, each quadrant $R$ is associated to its symmetric quadrant $R^{*}$ to obtain $Q=$ $2^{n_{x}+n_{u}+n_{w}-1}$ regions:

$$
R_{q}\left(s_{1}^{(x)}, \ldots, s_{j}^{(u)}, \ldots, s_{n_{w}}^{(w)}\right) \cup R_{q}^{*}\left(\neg s_{1}^{(x)}, \ldots, \neg s_{j}^{(u)}, \ldots, \neg s_{n_{w}}^{(w)}\right)
$$

where $\neg$ denotes the negation operator and $q=1, \ldots, Q$.

- In the second step, for each of the regions $R_{q} \cup R_{q}^{*}, q=1, \ldots, Q$ defined in (45), after partially differentiating each row $f_{i}$ of (12) with respect to $x_{1}, \ldots, x_{n_{x}}$, $u_{1}, \ldots, u_{n_{u}}$, the minimum and maximum values in the region $R_{q} \cup R_{q}^{*}$ are found:

$$
\begin{aligned}
& \bar{a}_{i j}^{(q)}=\max _{x, u, w \in R_{q} \cup R_{q}^{*}} \frac{\partial f_{i}(x, u, w)}{\partial x_{j}} \quad \begin{array}{l}
i=1, \ldots, n_{x} \\
j=1, \ldots, n_{x}
\end{array} \\
& \underline{a}_{i j}^{(q)}=\min _{x, u, w \in R_{q} \cup R_{q}^{*}} \frac{\partial f_{i}(x, u, w)}{\partial x_{j}} \quad \begin{array}{l}
i=1, \ldots, n_{x} \\
j=1, \ldots, n_{x}
\end{array} \\
& \bar{b}_{i j}^{(q)}=\max _{x, u, w \in R_{q} \cup R_{q}^{*}} \frac{\partial f_{i}(x, u, w)}{\partial u_{j}} \quad \begin{array}{l}
i=1, \ldots, n_{x} \\
j=1, \ldots, n_{u}
\end{array} \\
& \underline{b}_{i j}^{(q)}=\min _{x, u, w \in R_{q} \cup R_{q}^{*}} \frac{\partial f_{i}(x, u, w)}{\partial u_{j}} \quad \begin{array}{l}
i=1, \ldots, n_{x} \\
j=1, \ldots, n_{u}
\end{array}
\end{aligned}
$$

- In the third step, the vertex matrices $\left(A_{j}^{(q)}, B_{j}^{(q)}\right)$ are obtained by taking into consideration all the possible combinations of the row vectors $\left[\hat{a}_{i}^{(q)}, \hat{b}_{i}^{(q)}\right]$ and $\left[\breve{a}_{i}^{(q)}, \breve{b}_{i}^{(q)}\right]$, as follows: 


$$
\begin{aligned}
A_{j}^{(q)}\left(t_{1}^{(j)}, \ldots, t_{i}^{(j)}, \ldots, t_{n_{x}}^{(j)}\right) & =\left(\begin{array}{c}
\tilde{a}_{1}^{(q)} \\
\vdots \\
\tilde{a}_{i}^{(q)} \\
\vdots \\
\tilde{a}_{n_{x}}^{(q)}
\end{array}\right) \\
B_{j}^{(q)}\left(t_{1}^{(j)}, \ldots, t_{i}^{(j)}, \ldots, t_{n_{x}}^{(j)}\right) & =\left(\begin{array}{c}
\tilde{b}_{1}^{(q)} \\
\vdots \\
\tilde{b}_{i}^{(q)} \\
\vdots \\
\tilde{b}_{n_{x}}^{(q)}
\end{array}\right)
\end{aligned}
$$

where:

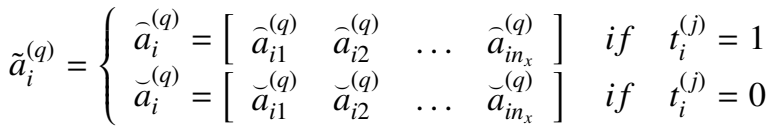

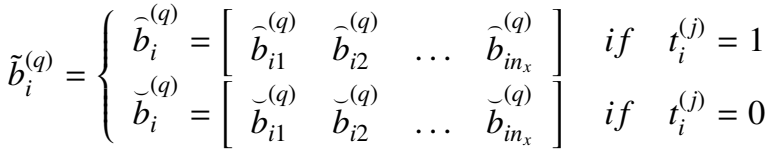

and:

$$
\begin{aligned}
& \hat{a}_{i j}^{(q)}=\left\{\begin{array}{lll}
\bar{a}_{i j}^{(q)} & \text { if } & s_{j}^{(x)}(q)=1 \\
\underline{a}_{i j}^{(q)} & \text { if } & s_{j}^{(x)}(q)=0
\end{array}\right. \\
& \widetilde{a}_{i j}^{(q)}=\left\{\begin{array}{lll}
\underline{a}_{i j}^{(q)} & \text { if } & s_{j}^{(x)}(q)=1 \\
\bar{a}_{i j}^{(q)} & \text { if } & s_{j}^{(x)}(q)=0
\end{array}\right. \\
& \hat{b}_{i j}^{(q)}=\left\{\begin{array}{lll}
\bar{b}_{i j}^{(q)} & \text { if } & s_{j}^{(u)}(q)=1 \\
\underline{b}_{i j}^{(q)} & \text { if } & s_{j}^{(u)}(q)=0
\end{array}\right. \\
& \breve{b}_{i j}^{(q)}=\left\{\begin{array}{lll}
\underline{b}_{i j}^{(q)} & \text { if } & s_{j}^{(u)}(q)=1 \\
\bar{b}_{i j}^{(q)} & \text { if } & s_{j}^{(u)}(q)=0
\end{array}\right.
\end{aligned}
$$

Then, (12) can be reconstructed from $\left(A_{j}^{(q)}, B_{j}^{(q)}\right)$ as follows:

$$
\sigma . x=f(x, u, w)=\sum_{q=1}^{Q} \sum_{j=1}^{2^{n_{x}}} \alpha_{j}^{(q)}(x, u, w)\left(A_{j}^{(q)} x+B_{j}^{(q)} u\right)
$$

where:

$$
\alpha_{j}^{(q)}(x, u, w)=\prod_{i=1}^{n_{x}}\left[t_{i}^{(j)} \widehat{\alpha}_{i}^{(q)}(x, u, w)+\left(1-t_{i}^{(j)}\right) \breve{\alpha}_{i}^{(q)}(x, u, w)\right]
$$


with:

$$
\begin{aligned}
& \hat{\alpha}_{i}^{(q)}(x, u, w)=\frac{f_{i}(x, u, w)-\widehat{a}_{i}^{(q)} x-\breve{b}_{i}^{(q)} u}{\hat{a}_{i}^{(q)} x+\widehat{b}_{i}^{(q)} u-\widehat{a}_{i}^{(q)} x-\breve{b}_{i}^{(q)} u} R_{q}^{\epsilon}(x, u, w) \\
& \breve{\alpha}_{i}^{(q)}(x, u, w)=\frac{\widehat{a}_{i}^{(q)} x+\widehat{b}_{i}^{(q)} u-f_{i}(x, u, w)}{\hat{a}_{i}^{(q)} x+\widehat{b}_{i}^{(q)} u-\widehat{a}_{i}^{(q)} x-\breve{b}_{i}^{(q)} u} R_{q}^{\epsilon}(x, u, w)
\end{aligned}
$$

where $R_{q}^{\epsilon}(x, u, w)$ is an operator that returns 1 if $(x, u, w)$ belongs to the region $R_{q} \cup R_{q}^{*}$ and 0 otherwise.

Remark 1: Notice that the polytopic system (58) is equivalent to the following quasi-LPV system:

$$
\sigma \cdot x=A(x, u, w) x+B(x, u, w) u
$$

with:

$$
\begin{aligned}
& A(x, u, w)=\sum_{q=1}^{Q} \sum_{j=1}^{2^{n_{x}}} \alpha_{j}^{(q)}(x, u, w) A_{j}^{(q)} \\
& B(x, u, w)=\sum_{q=1}^{Q} \sum_{j=1}^{2_{x}} \alpha_{j}^{(q)}(x, u, w) B_{j}^{(q)}
\end{aligned}
$$

Remark 2: The obtained polytopic system exhibits discontinuities in the polytopic decomposition coefficients $\alpha_{j}^{(q)}(x, u, w)$ at the region boundaries, i.e. along the axes that define the quadrants. In order to avoid this phenomenon, [31] suggests to add some compatibility conditions. In particular, this is obtained by replacing $\bar{a}_{i j}^{(q)}, \underline{a}_{i j}^{(q)}, \bar{b}_{i j}^{(q)}$ and $\underline{b}_{i j}^{(q)}$ in (54)-(57) with $\bar{a}_{i j}, \underline{a}_{i j}, \bar{b}_{i j}, \underline{b}_{i j}$, defined as follows:

$$
\begin{aligned}
\bar{a}_{i j}=\max _{q=1, \ldots, Q} \bar{a}_{i j}^{(q)} & i=1, \ldots, n_{x} \\
\underline{a}_{i j}=\min _{q=1, \ldots, Q} \underline{a}_{i j}^{(q)} & i=1, \ldots, n_{x} \\
\bar{b}_{i j}=\max _{q=1, \ldots, Q} \bar{b}_{i j}^{(q)} & i=1, \ldots, n_{x} \\
\underline{b}_{i j}=\min _{q=1, \ldots, Q} \underline{b}_{i j}^{(q)} & i=1, \ldots, n_{x} \\
& j=1, \ldots, n_{x} \\
& j=1, \ldots, n_{u}
\end{aligned}
$$

An example of the application of the proposed technique is illustrated in Section 6.2 .

\section{Application example}

Consider the following non-linear system: 


$$
\left\{\begin{array}{l}
\dot{x}_{1}=x_{1}+3 \sin x_{1}+x_{2}-2 \sin x_{2}+u_{1} \\
\dot{x}_{2}=x_{1}^{2} \sqrt{1+x_{2}^{2}}+x_{1} x_{2}+u_{2} \\
\dot{x}_{3}=x_{1}+x_{2}-x_{3}
\end{array}\right.
$$

with:

$$
x_{1}, x_{2}, x_{3} \in \mathcal{P}=[-\pi, \pi] \times[-\pi, \pi] \times[-\pi, \pi]
$$

The aim of this section is to obtain a TS and a quasi-LPV representation of (69) using the methods described in Sections 4 and 5.

\subsection{Generation of TS models via non-linear embedding}

The TS representations are obtained applying the non-linear embedding method described in Section 4, where the final step is done by superposition, such that eight different TS models are generated. The general form for each TS model is the following:

$$
\begin{gathered}
\text { IF } \vartheta_{11}^{(j)} \text { is } M_{i 11}^{(j)} \text { AND } \vartheta_{12}^{(j)} \text { is } M_{i 12}^{(j)} A N D \vartheta_{21}^{(j)} \text { is } M_{i 21}^{(j)} \text { AND } \vartheta_{22}^{(j)} \text { is } M_{i 22}^{(j)} \\
\text { THEN } \quad \dot{x}(t)=A_{i}^{(j)} x(t)+\left(\begin{array}{cc}
1 & 0 \\
0 & 1 \\
0 & 0
\end{array}\right) u(t) \\
i=1, \ldots, N_{j} \\
j=1, \ldots, 8
\end{gathered}
$$

where for the $j^{\text {th }}$ TS model, the $N_{j} \in\{4,8,16\}$ linear models are obtained taking into consideration all possible combinations of minimum and maximum values of the premise variables $\vartheta_{11}^{(j)}, \vartheta_{12}^{(j)}, \vartheta_{21}^{(j)}$ and $\vartheta_{22}^{(j)}$.

In particular, the premise variables are defined as follows ${ }^{3}$ :

$$
\begin{gathered}
\vartheta_{11}^{(1)}\left(x_{1}, x_{2}\right)=\vartheta_{11}^{(2)}\left(x_{1}, x_{2}\right)=1+3 \frac{\sin x_{1}}{x_{1}}-2 \frac{\sin x_{2}}{x_{1}} \\
\vartheta_{11}^{(3)}\left(x_{1}\right)=\vartheta_{11}^{(4)}\left(x_{1}\right)=1+3 \frac{\sin x_{1}}{x_{1}} \\
\vartheta_{11}^{(5)}\left(x_{1}, x_{2}\right)=\vartheta_{11}^{(6)}\left(x_{1}, x_{2}\right)=1-2 \frac{\sin x_{2}}{x_{1}} \\
\vartheta_{12}^{(3)}\left(x_{2}\right)=\vartheta_{12}^{(4)}\left(x_{2}\right)=1-2 \frac{\sin x_{2}}{x_{2}} \\
\vartheta_{12}^{(5)}\left(x_{1}, x_{2}\right)=\vartheta_{12}^{(6)}\left(x_{1}, x_{2}\right)=1+3 \frac{\sin x_{1}}{x_{2}} \\
\vartheta_{12}^{(7)}\left(x_{1}, x_{2}\right)=\vartheta_{12}^{(8)}\left(x_{1}, x_{2}\right)=1+3 \frac{\sin x_{1}}{x_{2}}-2 \frac{\sin x_{2}}{x_{2}} \\
\vartheta_{21}^{(1)}\left(x_{1}, x_{2}\right)=\vartheta_{21}^{(3)}\left(x_{1}, x_{2}\right)=\vartheta_{21}^{(5)}\left(x_{1}, x_{2}\right)=\vartheta_{21}^{(7)}\left(x_{1}, x_{2}\right)=x_{1} \sqrt{1+x_{2}^{2}}+x_{2}
\end{gathered}
$$

\footnotetext{
${ }^{3}$ Notice that the real premise variables can be a subset of those listed in (70), when some of them are constants, i.e. $\vartheta_{12}^{(1)}=\vartheta_{12}^{(2)}=\vartheta_{11}^{(7)}=\vartheta_{11}^{(8)}=1, \vartheta_{22}^{(1)}=\vartheta_{22}^{(3)}=\vartheta_{22}^{(5)}=\vartheta_{22}^{(7)}=0$.
} 


$$
\begin{gathered}
\vartheta_{21}^{(2)}\left(x_{1}, x_{2}\right)=\vartheta_{21}^{(4)}\left(x_{1}, x_{2}\right)=\vartheta_{21}^{(6)}\left(x_{1}, x_{2}\right)=\vartheta_{21}^{(8)}\left(x_{1}, x_{2}\right)=x_{1} \sqrt{1+x_{2}^{2}} \\
\vartheta_{22}^{(2)}\left(x_{1}\right)=\vartheta_{22}^{(4)}\left(x_{1}\right)=\vartheta_{22}^{(6)}\left(x_{1}\right)=\vartheta_{22}^{(8)}\left(x_{1}\right)=x_{1}
\end{gathered}
$$

Among the obtained models, the ones that are considered to be more suitable for representing the original non-linear system (69) are those given by $j=3$ and $j=4$. This is motivated by the fact that in the remaining six TS models, i.e. $j \in$ $\{1,2,5,6,7,8\}$, terms of the type $\sin x_{1} / x_{2}$ or $\sin x_{2} / x_{1}$ appear, which are not defined in some subsets of the region $\mathcal{P}$.

For the models obtained with $j=3$ and $j=4$, the subsystems in (70) are defined by the following state matrices (see Fig. 1 for a graphical example of how a non-linear system equation is described by the subsystems):

$$
\begin{aligned}
& A_{1}^{(3)}=\left(\begin{array}{ccc}
4 & 1 & 0 \\
k_{\pi} & 0 & 0 \\
1 & 1 & -1
\end{array}\right) A_{2}^{(3)}=\left(\begin{array}{ccc}
4 & -1 & 0 \\
k_{\pi} & 0 & 0 \\
1 & 1 & -1
\end{array}\right) A_{3}^{(3)}=\left(\begin{array}{ccc}
4 & 1 & 0 \\
-k_{\pi} & 0 & 0 \\
1 & 1 & -1
\end{array}\right) \\
& A_{4}^{(3)}=\left(\begin{array}{ccc}
4 & -1 & 0 \\
-k_{\pi} & 0 & 0 \\
1 & 1 & -1
\end{array}\right) A_{5}^{(3)}=\left(\begin{array}{ccc}
1 & 1 & 0 \\
k_{\pi} & 0 & 0 \\
1 & 1 & -1
\end{array}\right) A_{6}^{(3)}=\left(\begin{array}{ccc}
1 & -1 & 0 \\
k_{\pi} & 0 & 0 \\
1 & 1 & -1
\end{array}\right) \\
& A_{7}^{(3)}=\left(\begin{array}{ccc}
1 & 1 & 0 \\
-k_{\pi} & 0 & 0 \\
1 & 1 & -1
\end{array}\right) A_{8}^{(3)}=\left(\begin{array}{ccc}
1 & -1 & 0 \\
-k_{\pi} & 0 & 0 \\
1 & 1 & -1
\end{array}\right) A_{1}^{(4)}=\left(\begin{array}{ccc}
4 & 1 & 0 \\
q_{\pi} & \pi & 0 \\
1 & 1 & -1
\end{array}\right) \\
& A_{2}^{(4)}=\left(\begin{array}{ccc}
4 & 1 & 0 \\
q_{\pi} & -\pi & 0 \\
1 & 1 & -1
\end{array}\right) A_{3}^{(4)}=\left(\begin{array}{ccc}
4 & 1 & 0 \\
-q_{\pi} & \pi & 0 \\
1 & 1 & -1
\end{array}\right) A_{4}^{(4)}=\left(\begin{array}{ccc}
4 & 1 & 0 \\
-q_{\pi} & -\pi & 0 \\
1 & 1 & -1
\end{array}\right) \\
& A_{5}^{(4)}=\left(\begin{array}{ccc}
4 & -1 & 0 \\
q_{\pi} & \pi & 0 \\
1 & 1 & -1
\end{array}\right) A_{6}^{(4)}=\left(\begin{array}{ccc}
4 & -1 & 0 \\
q_{\pi} & -\pi & 0 \\
1 & 1 & -1
\end{array}\right) A_{7}^{(4)}=\left(\begin{array}{ccc}
4 & -1 & 0 \\
-q_{\pi} & \pi & 0 \\
1 & 1 & -1
\end{array}\right) \\
& A_{8}^{(4)}=\left(\begin{array}{ccc}
4 & -1 & 0 \\
-q_{\pi} & -\pi & 0 \\
1 & 1 & -1
\end{array}\right) A_{9}^{(4)}=\left(\begin{array}{ccc}
1 & 1 & 0 \\
q_{\pi} & \pi & 0 \\
1 & 1 & -1
\end{array}\right) A_{10}^{(4)}=\left(\begin{array}{ccc}
1 & 1 & 0 \\
q_{\pi} & -\pi & 0 \\
1 & 1 & -1
\end{array}\right) \\
& A_{11}^{(4)}=\left(\begin{array}{ccc}
1 & 1 & 0 \\
-q_{\pi} & \pi & 0 \\
1 & 1 & -1
\end{array}\right) A_{12}^{(4)}=\left(\begin{array}{ccc}
1 & 1 & 0 \\
-q_{\pi} & -\pi & 0 \\
1 & 1 & -1
\end{array}\right) A_{13}^{(4)}=\left(\begin{array}{ccc}
1 & -1 & 0 \\
q_{\pi} & \pi & 0 \\
1 & 1 & -1
\end{array}\right) \\
& A_{14}^{(4)}=\left(\begin{array}{ccc}
1 & -1 & 0 \\
1 & -1 & 0 \\
q_{\pi} & -\pi & 0 \\
1 & 1 & -1
\end{array}\right) A_{15}^{(4)}=\left(\begin{array}{ccc}
1 & -1 & 0 \\
-q_{\pi} & \pi & 0 \\
1 & 1 & -1
\end{array}\right) A_{16}^{(4)}=\left(\begin{array}{ccc}
-\pi & 0 \\
-q_{\pi} & -\pi & 1 \\
1 & -1
\end{array}\right)
\end{aligned}
$$

where $k_{\pi}$ and $q_{\pi}$ are constants defined as:

$$
k_{\pi}=\pi \sqrt{1+\pi^{2}}+\pi \quad q_{\pi}=\pi \sqrt{1+\pi^{2}}
$$


The membership functions $M_{i 11}^{(3)}, M_{i 12}^{(3)}, M_{i 21}^{(3)}, M_{i 11}^{(4)}, M_{i 12}^{(4)}, M_{i 21}^{(4)}, M_{i 22}^{(4)}$ are defined using (40):

$$
\begin{gathered}
M_{i 11}^{(3)}\left(\vartheta_{11}^{(3)}\left(x_{1}\right)\right)= \begin{cases}\sin x_{1} / x_{1} & i=1,2,3,4 \\
1-\sin x_{1} / x_{1} & i=5,6,7,8\end{cases} \\
M_{i 12}^{(3)}\left(\vartheta_{12}^{(3)}\left(x_{2}\right)\right)= \begin{cases}1-\sin x_{2} / x_{2} & i=1,3,5,7 \\
\sin x_{2} / x_{2} & i=2,4,6,8\end{cases} \\
M_{i 21}^{(3)}\left(\vartheta_{21}^{(3)}\left(x_{1}, x_{2}\right)\right)= \begin{cases}\frac{x_{1} \sqrt{1+x_{2}^{2}}+x_{2}+k_{\pi}}{2 k_{\pi}} & i=1,2,5,6 \\
\frac{k_{\pi}-x_{1} \sqrt{1+x_{2}^{2}}-x_{2}}{2 k_{\pi}} & i=3,4,7,8\end{cases} \\
M_{i 11}^{(4)}\left(\vartheta_{11}^{(4)}\left(x_{1}\right)\right)= \begin{cases}\sin x_{1} / x_{1} & i=1,2,3,4,5,6,7,8 \\
1-\sin x_{1} / x_{1} & i=9,10,11,12,13,14,15,16\end{cases} \\
M_{i 12}^{(4)}\left(\vartheta_{12}^{(4)}\left(x_{2}\right)\right)= \begin{cases}1-\sin x_{2} / x_{2} & i=1,2,3,4,9,10,11,12 \\
\sin x_{2} / x_{2} & i=5,6,7,8,13,14,15,16\end{cases} \\
M_{i 21}^{(4)}\left(\vartheta_{21}^{(4)}\left(x_{1}, x_{2}\right)\right)= \begin{cases}\frac{x_{1} \sqrt{1+x_{2}^{2}}+q_{\pi}}{2 q_{\pi}} & i=1,2,5,6,9,10,13,14 \\
\frac{q_{\pi}-x_{1} \sqrt{1+x_{2}^{2}}}{2 q_{\pi}} & i=3,4,7,8,11,12,15,16\end{cases} \\
M_{i 22}^{(4)}\left(\vartheta_{22}^{(4)}\left(x_{1}\right)\right)= \begin{cases}\frac{x_{1}+\pi}{2 \pi} & i=1,3,5,7,9,11,13,15 \\
\frac{\pi-x_{1}}{2 \pi} & i=2,4,6,8,10,12,14,16\end{cases}
\end{gathered}
$$

Finally, the coefficients that describe the level of activation of each local model are obtained using (10) as:

$$
\begin{gathered}
\rho_{i}^{(3)}\left(x_{1}, x_{2}\right)=\frac{M_{i 11}^{(3)} M_{i 12}^{(3)} M_{i 21}^{(3)}}{\sum_{i=1}^{8} M_{i 11}^{(3)} M_{i 12}^{(3)} M_{i 21}^{(3)}} \\
\rho_{i}^{(4)}\left(x_{1}, x_{2}\right)=\frac{M_{i 11}^{(4)} M_{i 12}^{(4)} M_{i 21}^{(4)} M_{i 22}^{(4)}}{\sum_{i=1}^{16} M_{i 11}^{(4)} M_{i 12}^{(4)} M_{i 21}^{(4)} M_{i 22}^{(4)}}
\end{gathered}
$$

Remark 3: Notice that the obtained TS models can be interpreted as if they were LPV systems as follows:

$$
\begin{aligned}
& \left(\begin{array}{l}
\dot{x}_{1} \\
\dot{x}_{2} \\
\dot{x}_{3}
\end{array}\right)=A_{3}\left(x_{1}, x_{2}\right)\left(\begin{array}{l}
x_{1} \\
x_{2} \\
x_{3}
\end{array}\right)+\left(\begin{array}{ll}
1 & 0 \\
0 & 1 \\
0 & 0
\end{array}\right)\left(\begin{array}{l}
u_{1} \\
u_{2}
\end{array}\right) \\
& \left(\begin{array}{l}
\dot{x}_{1} \\
\dot{x}_{2} \\
\dot{x}_{3}
\end{array}\right)=A_{4}\left(x_{1}, x_{2}\right)\left(\begin{array}{l}
x_{1} \\
x_{2} \\
x_{3}
\end{array}\right)+\left(\begin{array}{ll}
1 & 0 \\
0 & 1 \\
0 & 0
\end{array}\right)\left(\begin{array}{l}
u_{1} \\
u_{2}
\end{array}\right)
\end{aligned}
$$


where:

$$
\begin{aligned}
& A_{3}\left(x_{1}, x_{2}\right)=\left(\begin{array}{ccc}
\vartheta_{11}^{(3)}\left(x_{1}\right) & \vartheta_{12}^{(3)}\left(x_{2}\right) & 0 \\
\vartheta_{21}^{(3)}\left(x_{1}, x_{2}\right) & 0 & 0 \\
1 & 1 & -1
\end{array}\right)=\sum_{i=1}^{8} \rho_{i}^{(3)}\left(x_{1}, x_{2}\right) A_{i}^{(3)} \\
& A_{4}\left(x_{1}, x_{2}\right)=\left(\begin{array}{ccc}
\vartheta_{11}^{(4)}\left(x_{1}\right) & \vartheta_{12}^{(4)}\left(x_{2}\right) & 0 \\
\vartheta_{21}^{(4)}\left(x_{1}, x_{2}\right) & \vartheta_{22}^{(4)}\left(x_{1}\right) & 0 \\
1 & 1 & -1
\end{array}\right)=\sum_{i=1}^{16} \rho_{i}^{(4)}\left(x_{1}, x_{2}\right) A_{i}^{(4)}
\end{aligned}
$$

where $\rho_{i}^{(3)}\left(x_{1}, x_{2}\right)$ and $\rho_{i}^{(4)}\left(x_{1}, x_{2}\right)$ can be interpreted as coefficients of a polytopic decomposition.

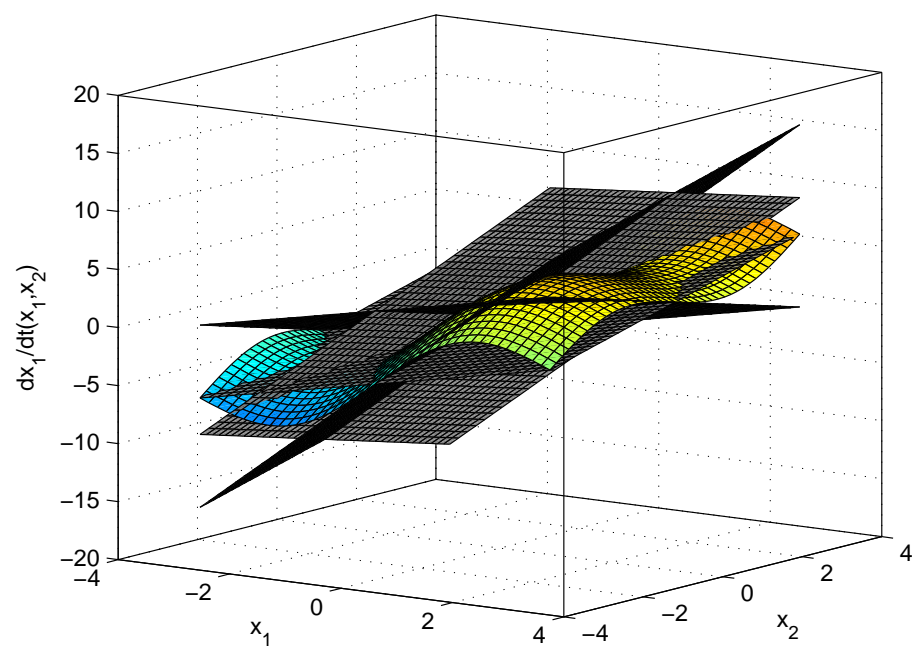

Figure 1: Representation of the non-linear equation $\dot{x}_{1}=x_{1}+3 \sin x_{1}+x_{2}-2 \sin x_{2}$ in $\mathcal{P}$ and its approximation using the subsystems described by $A_{i}^{(3)}$ or $A_{i}^{(4)}$.

\subsection{Generation of polytopic LPV models via sector non-linearity concept}

Hereafter, a polytopic representation for (69) is obtained applying the method described in Section 5.

The space $\left\{\mathbb{X}_{1}, \mathbb{X}_{2}\right\}$ is partitioned into 4 quadrants, that give rise to the following 2 regions as described by (45):

$$
\begin{aligned}
& R_{1}:[-\pi, 0] \times[-\pi, 0] \cup[0, \pi] \times[0, \pi] \\
& R_{2}:[-\pi, 0] \times[0, \pi] \cup[0, \pi] \times[-\pi, 0]
\end{aligned}
$$

Then, the partial derivatives of (69) are calculated:

$$
\frac{\partial f_{1}}{\partial x_{1}}=1+3 \cos x_{1} \quad \frac{\partial f_{1}}{\partial x_{2}}=1-2 \cos x_{2}
$$




$$
\frac{\partial f_{2}}{\partial x_{1}}=2 x_{1} \sqrt{1+x_{2}^{2}}+x_{2} \quad \frac{\partial f_{2}}{\partial x_{2}}=\frac{x_{1}^{2} x_{2}}{\sqrt{1+x_{2}^{2}}}+x_{1}
$$

and their minimum and maximum values in $R_{1}$ and $R_{2}$ are found:

$$
\begin{array}{cll}
\bar{a}_{11}^{(1)}=\max _{R_{1}} \frac{\partial f_{1}}{\partial x_{1}}=4 & \underline{a}_{11}^{(1)}=\min _{R_{1}} \frac{\partial f_{1}}{\partial x_{1}}=-2 \\
\bar{a}_{11}^{(2)}=\max _{R_{2}} \frac{\partial f_{1}}{\partial x_{1}}=4 & \underline{a}_{11}^{(2)}=\min _{R_{2}} \frac{\partial f_{1}}{\partial x_{1}}=-2 \\
\bar{a}_{12}^{(1)}=\max _{R_{1}} \frac{\partial f_{1}}{\partial x_{2}}=3 & \underline{a}_{12}^{(1)}=\min _{R_{1}} \frac{\partial f_{1}}{\partial x_{2}}=-1 \\
\bar{a}_{12}^{(2)}=\max _{R_{2}} \frac{\partial f_{1}}{\partial x_{2}}=3 & \underline{a}_{12}^{(2)}=\min _{R_{2}} \frac{\partial f_{1}}{\partial x_{2}}=-1 \\
\bar{a}_{21}^{(1)}=\max _{R_{1}} \frac{\partial f_{2}}{\partial x_{1}}=r_{\pi}+\pi & \underline{a}_{21}^{(1)}=\min _{R_{1}} \frac{\partial f_{2}}{\partial x_{1}}=-\left(r_{\pi}+\pi\right) \\
\bar{a}_{21}^{(2)}=\max _{R_{2}} \frac{\partial f_{2}}{\partial x_{1}}=r_{\pi}-\pi & \underline{a}_{21}^{(2)}=\min _{R_{2}} \frac{\partial f_{2}}{\partial x_{1}}=-r_{\pi}+\pi \\
\bar{a}_{22}^{(1)}=\max _{R_{1}} \frac{\partial f_{2}}{\partial x_{2}}=w_{\pi}+\pi & \underline{a}_{22}^{(1)}=\min _{R_{1}} \frac{\partial f_{2}}{\partial x_{2}}=-\left(w_{\pi}+\pi\right) \\
\bar{a}_{22}^{(2)}=\max _{R_{2}} \frac{\partial f_{2}}{\partial x_{2}}=w_{\pi}-\pi & \underline{a}_{22}^{(2)}=\min _{R_{2}} \frac{\partial f_{2}}{\partial x_{2}}=-w_{\pi}+\pi
\end{array}
$$

where:

$$
r_{\pi}=2 \pi \sqrt{1+\pi^{2}} \quad w_{\pi}=\frac{\pi^{3}}{\sqrt{1+\pi^{2}}}
$$

Afterwards, using (50)-(57), the state matrices of the vertex systems are calculated, resulting in the following eight matrices (see Fig. 2 for a graphical representation example):

$$
\begin{aligned}
& A_{1}^{(1)}=\left(\begin{array}{ccc}
-2 & -1 & 0 \\
-\left(r_{\pi}+\pi\right) & -\left(w_{\pi}+\pi\right) & 0 \\
1 & 1 & -1
\end{array}\right) \quad A_{2}^{(1)}=\left(\begin{array}{ccc}
-2 & -1 & 0 \\
r_{\pi}+\pi & w_{\pi}+\pi & 0 \\
1 & 1 & -1
\end{array}\right) \\
& A_{3}^{(1)}=\left(\begin{array}{ccc}
4 & 3 & 0 \\
-\left(r_{\pi}+\pi\right) & -\left(w_{\pi}+\pi\right) & 0 \\
1 & 1 & -1
\end{array}\right) \quad A_{4}^{(1)}=\left(\begin{array}{ccc}
4 & 3 & 0 \\
r_{\pi}+\pi & w_{\pi}+\pi & 0 \\
1 & 1 & -1
\end{array}\right) \\
& A_{1}^{(2)}=\left(\begin{array}{ccc}
-2 & -1 & 0 \\
-r_{\pi}+\pi & w_{\pi}-\pi & 0 \\
1 & 1 & -1
\end{array}\right) \quad A_{2}^{(2)}=\left(\begin{array}{ccc}
-2 & -1 & 0 \\
r_{\pi}-\pi & -w_{\pi}+\pi & 0 \\
1 & 1 & -1
\end{array}\right) \\
& A_{3}^{(2)}=\left(\begin{array}{ccc}
4 & 3 & 0 \\
-r_{\pi}+\pi & w_{\pi}-\pi & 0 \\
1 & 1 & -1
\end{array}\right) \quad A_{4}^{(2)}=\left(\begin{array}{ccc}
4 & 3 & 0 \\
r_{\pi}-\pi & -w_{\pi}+\pi & 0 \\
1 & 1 & -1
\end{array}\right)
\end{aligned}
$$


such that (69) results expressed in the following polytopic LPV form:

$$
\left(\begin{array}{c}
\dot{x}_{1} \\
\dot{x}_{2} \\
\dot{x}_{3}
\end{array}\right)=\sum_{j=1}^{4} \alpha_{j}^{(1)}\left(x_{1}, x_{2}\right) A_{j}^{(1)}\left(\begin{array}{c}
x_{1} \\
x_{2} \\
x_{3}
\end{array}\right)+\sum_{j=1}^{4} \alpha_{j}^{(2)}\left(x_{1}, x_{2}\right) A_{j}^{(2)}\left(\begin{array}{c}
x_{1} \\
x_{2} \\
x_{3}
\end{array}\right)+\left(\begin{array}{ll}
1 & 0 \\
0 & 1 \\
0 & 0
\end{array}\right)\left(\begin{array}{l}
u_{1} \\
u_{2}
\end{array}\right)
$$

where the coefficients of the polytopic decomposition are obtained using (59)-(61), as follows:

$$
\begin{aligned}
& \alpha_{1}^{(1)}\left(x_{1}, x_{2}\right)=\widehat{\alpha}_{1}^{(1)}\left(x_{1}, x_{2}\right) \widehat{\alpha}_{2}^{(1)}\left(x_{1}, x_{2}\right) R_{1}^{\epsilon}\left(x_{1}, x_{2}\right) \\
& \alpha_{2}^{(1)}\left(x_{1}, x_{2}\right)=\widehat{\alpha}_{1}^{(1)}\left(x_{1}, x_{2}\right) \breve{\alpha}_{2}^{(1)}\left(x_{1}, x_{2}\right) R_{1}^{\epsilon}\left(x_{1}, x_{2}\right) \\
& \alpha_{3}^{(1)}\left(x_{1}, x_{2}\right)=\widetilde{\alpha}_{1}^{(1)}\left(x_{1}, x_{2}\right) \widehat{\alpha}_{2}^{(1)}\left(x_{1}, x_{2}\right) R_{1}^{\epsilon}\left(x_{1}, x_{2}\right) \\
& \alpha_{4}^{(1)}\left(x_{1}, x_{2}\right)=\widetilde{\alpha}_{1}^{(1)}\left(x_{1}, x_{2}\right) \widehat{\alpha}_{2}^{(1)}\left(x_{1}, x_{2}\right) R_{1}^{\epsilon}\left(x_{1}, x_{2}\right) \\
& \alpha_{1}^{(2)}\left(x_{1}, x_{2}\right)=\widehat{\alpha}_{1}^{(2)}\left(x_{1}, x_{2}\right) \widehat{\alpha}_{2}^{(2)}\left(x_{1}, x_{2}\right) R_{2}^{\epsilon}\left(x_{1}, x_{2}\right) \\
& \alpha_{2}^{(2)}\left(x_{1}, x_{2}\right)=\widehat{\alpha}_{1}^{(2)}\left(x_{1}, x_{2}\right) \widehat{\alpha}_{2}^{(2)}\left(x_{1}, x_{2}\right) R_{2}^{\epsilon}\left(x_{1}, x_{2}\right) \\
& \alpha_{3}^{(2)}\left(x_{1}, x_{2}\right)=\widetilde{\alpha}_{1}^{(2)}\left(x_{1}, x_{2}\right) \widehat{\alpha}_{2}^{(2)}\left(x_{1}, x_{2}\right) R_{2}^{\epsilon}\left(x_{1}, x_{2}\right) \\
& \alpha_{4}^{(2)}\left(x_{1}, x_{2}\right)=\breve{\alpha}_{1}^{(2)}\left(x_{1}, x_{2}\right) \breve{\alpha}_{2}^{(2)}\left(x_{1}, x_{2}\right) R_{2}^{\epsilon}\left(x_{1}, x_{2}\right)
\end{aligned}
$$

with:

$$
\begin{gathered}
\hat{\alpha}_{1}^{(1)}\left(x_{1}, x_{2}\right)=\frac{3 x_{1}+2 x_{2}-3 \sin x_{1}+2 \sin x_{2}}{6 x_{1}+4 x_{2}} \\
\widehat{\alpha}_{1}^{(1)}\left(x_{1}, x_{2}\right)=\frac{3 x_{1}+2 x_{2}+3 \sin x_{1}-2 \sin x_{2}}{6 x_{1}+4 x_{2}} \\
\hat{\alpha}_{1}^{(2)}\left(x_{1}, x_{2}\right)=\frac{3 x_{1}-2 x_{2}-3 \sin x_{1}+2 \sin x_{2}}{6 x_{1}-4 x_{2}} \\
\widetilde{\alpha}_{1}^{(2)}\left(x_{1}, x_{2}\right)=\frac{3 x_{1}-2 x_{2}+3 \sin x_{1}-2 \sin x_{2}}{6 x_{1}-4 x_{2}} \\
\widehat{\alpha}_{2}^{(1)}\left(x_{1}, x_{2}\right)=\frac{\left(r_{\pi}+\pi\right) x_{1}+\left(w_{\pi}+\pi\right) x_{2}-x_{1}^{2} \sqrt{1+x_{2}^{2}}-x_{1} x_{2}}{2\left[\left(r_{\pi}+\pi\right) x_{1}+\left(w_{\pi}+\pi\right) x_{2}\right]} \\
\breve{\alpha}_{2}^{(1)}\left(x_{1}, x_{2}\right)=\frac{\left(r_{\pi}+\pi\right) x_{1}+\left(w_{\pi}+\pi\right) x_{2}+x_{1}^{2} \sqrt{1+x_{2}^{2}}+x_{1} x_{2}}{2\left[\left(r_{\pi}+\pi\right) x_{1}+\left(w_{\pi}+\pi\right) x_{2}\right]} \\
\hat{\alpha}_{2}^{(2)}\left(x_{1}, x_{2}\right)=\frac{\left(r_{\pi}-\pi\right) x_{1}+\left(\pi-w_{\pi}\right) x_{2}-x_{1}^{2} \sqrt{1+x_{2}^{2}}-x_{1} x_{2}}{2\left[\left(r_{\pi}-\pi\right) x_{1}+\left(\pi-w_{\pi}\right) x_{2}\right]} \\
\breve{\alpha}_{2}^{(2)}\left(x_{1}, x_{2}\right)=\frac{\left(r_{\pi}-\pi\right) x_{1}+\left(\pi-w_{\pi}\right) x_{2}+x_{1}^{2} \sqrt{1+x_{2}^{2}}+x_{1} x_{2}}{2\left[\left(r_{\pi}-\pi\right) x_{1}+\left(\pi-w_{\pi}\right) x_{2}\right]}
\end{gathered}
$$




$$
\begin{gathered}
R_{1}^{\epsilon}\left(x_{1}, x_{2}\right)=\max \left(0, \operatorname{sgn}\left(x_{1}\right) \operatorname{sgn}\left(x_{2}\right)\right) \\
R_{2}^{\epsilon}\left(x_{1}, x_{2}\right)=\max \left(0,-\operatorname{sgn}\left(x_{1}\right) \operatorname{sgn}\left(x_{2}\right)\right)
\end{gathered}
$$

where $s g n$ denotes the sign function.

Remark 4: Notice that the quasi-LPV representation of (69) obtained using this method has the following structure:

$$
\left(\begin{array}{c}
\dot{x}_{1} \\
\dot{x}_{2} \\
\dot{x}_{3}
\end{array}\right)=\left(\begin{array}{ccc}
a_{11}\left(x_{1}, x_{2}\right) & a_{12}\left(x_{1}, x_{2}\right) & 0 \\
a_{21}\left(x_{1}, x_{2}\right) & a_{22}\left(x_{1}, x_{2}\right) & 0 \\
1 & 1 & -1
\end{array}\right)\left(\begin{array}{c}
x_{1} \\
x_{2} \\
x_{3}
\end{array}\right)+\left(\begin{array}{cc}
1 & 0 \\
0 & 1 \\
0 & 0
\end{array}\right)\left(\begin{array}{l}
u_{1} \\
u_{2}
\end{array}\right)
$$

Remark 5: The obtained quasi-LPV system can be interpreted as a Takagi-Sugeno model, if $a_{11}\left(x_{1}, x_{2}\right), a_{12}\left(x_{1}, x_{2}\right), a_{21}\left(x_{1}, x_{2}\right), a_{22}\left(x_{1}, x_{2}\right)$ in (95) and $\operatorname{sgn}\left(x_{1}\right) \operatorname{sgn}\left(x_{2}\right)$ are considered to be the premise variables, and $\hat{\alpha}_{1}^{(1)}, \widehat{\alpha}_{1}^{(1)}, \widehat{\alpha}_{1}^{(2)}, \breve{\alpha}_{1}^{(2)}, \widehat{\alpha}_{2}^{(1)}, \vec{\alpha}_{2}^{(1)}, \widehat{\alpha}_{2}^{(2)}, \breve{\alpha}_{2}^{(2)}, R_{1}^{\epsilon}$, $R_{2}^{\epsilon}$ the membership functions.

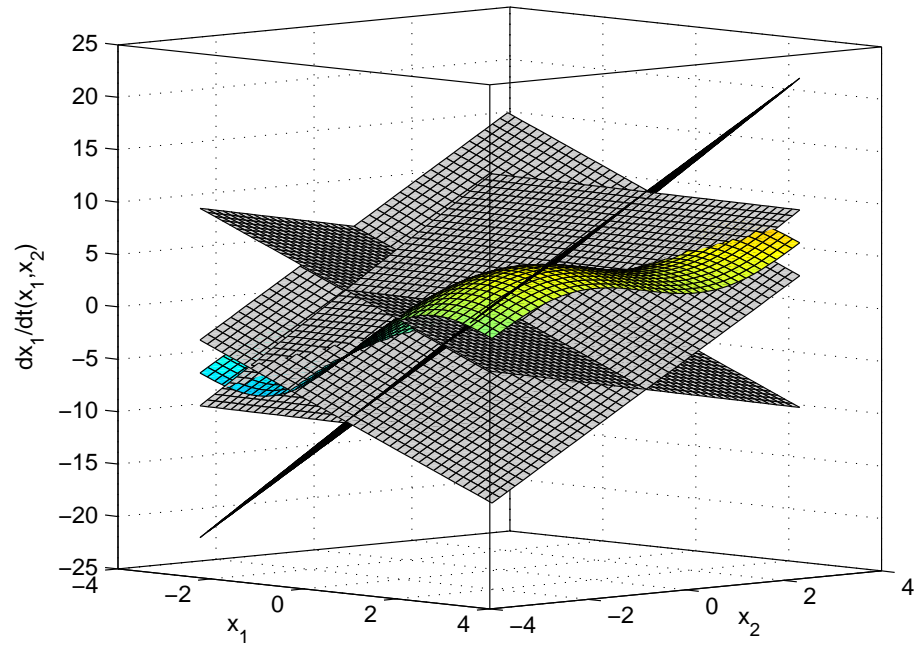

Figure 2: Representation of the non-linear equation $\dot{x}_{1}=x_{1}+3 \sin x_{1}+x_{2}-2 \sin x_{2}$ in $\mathcal{P}$ and its approximation using the vertex systems of (94).

Remark 6: If the conditions (65)-(68) are used in order to avoid the discontinuity phenomenon, as described in Section 5, the matrices $A_{1}^{(2)}, A_{2}^{(2)}, A_{3}^{(2)}$ and $A_{4}^{(2)}$ change as follows:

$$
A_{1}^{(2)}=\left(\begin{array}{ccc}
-2 & -1 & 0 \\
-\left(r_{\pi}+\pi\right) & w_{\pi}+\pi & 0 \\
1 & 1 & -1
\end{array}\right) \quad A_{2}^{(2)}=\left(\begin{array}{ccc}
-2 & -1 & 0 \\
r_{\pi}+\pi & -\left(w_{\pi}+\pi\right) & 0 \\
1 & 1 & -1
\end{array}\right)
$$




$$
A_{3}^{(2)}=\left(\begin{array}{ccc}
4 & 3 & 0 \\
-\left(r_{\pi}+\pi\right) & w_{\pi}+\pi & 0 \\
1 & 1 & -1
\end{array}\right) \quad A_{4}^{(2)}=\left(\begin{array}{ccc}
4 & 3 & 0 \\
r_{\pi}+\pi & -\left(w_{\pi}+\pi\right) & 0 \\
1 & 1 & -1
\end{array}\right)
$$

\subsection{Comparison}

Hereafter, the comparison criteria between models described in Section 3 are applied to the proposed example.

The subsets $S_{1} \subset \mathbb{X}_{1} \times \mathbb{X}_{2} \times \dot{\mathbb{X}}_{1}$ and $S_{2} \subset \mathbb{X}_{1} \times \mathbb{X}_{2} \times \dot{\mathbb{X}}_{2}$ are chosen as follows:

$$
\begin{aligned}
& S_{1}=[-\pi, \pi] \times[-\pi, \pi] \times[-7 \pi, 7 \pi] \\
& S_{2}=[-\pi, \pi] \times[-\pi, \pi] \times\left[-h_{\pi}, h_{\pi}\right]
\end{aligned}
$$

with:

$$
h_{\pi}=\pi^{2}\left(2+2 \sqrt{1+\pi^{2}}+\frac{\pi^{2}}{\sqrt{1+\pi^{2}}}\right)
$$

so that:

$$
V_{1}^{(S)}=56 \pi^{3} \quad V_{2}^{(S)}=8 \pi^{2} h_{\pi}
$$

The volumes $\tilde{V}_{i}$ have been calculated using (15) on the basis of $N=16588$ points $^{4}$, generated randomly using a uniform distribution:

Model generated via non-linear embedding $A_{i}^{(3)}$ :

$\frac{\tilde{V}_{1}}{V_{1}^{(S)}}=\frac{5856+3.3179}{16588+6.6358}=0.35 \quad \frac{\tilde{V}_{2}}{V_{2}^{(S)}}=\frac{6178+3.3179}{16588+6.6358}=0.37 \quad \tilde{M}=\frac{\tilde{V}_{1} \tilde{V}_{2}}{V_{1}^{(S)} V_{2}^{(S)}}=0.13$

Model generated via non-linear embedding $A_{i}^{(4)}$ :

$\frac{\tilde{V}_{1}}{V_{1}^{(S)}}=\frac{5856+3.3179}{16588+6.6358}=0.35 \quad \frac{\tilde{V}_{2}}{V_{2}^{(S)}}=\frac{4866+3.3179}{16588+6.6358}=0.29 \quad \tilde{M}=\frac{\tilde{V}_{1} \tilde{V}_{2}}{V_{1}^{(S)} V_{2}^{(S)}}=0.10$

Model generated via sector non-linearity concept:

$\frac{\tilde{V}_{1}}{V_{1}^{(S)}}=\frac{7546+3.3179}{16588+6.6358}=0.45 \quad \frac{\tilde{V}_{2}}{V_{2}^{(S)}}=\frac{9844+3.3179}{16588+6.6358}=0.59 \quad \tilde{M}=\frac{\tilde{V}_{1} \tilde{V}_{2}}{V_{1}^{(S)} V_{2}^{(S)}}=0.27$

Model generated via sector non-linearity concept (conservative):

$\frac{\tilde{V}_{1}}{V_{1}^{(S)}}=\frac{7546+3.3179}{16588+6.6358}=0.45 \quad \frac{\tilde{V}_{2}}{V_{2}^{(S)}}=\frac{11197+3.3179}{16588+6.6358}=0.67 \quad \tilde{M}=\frac{\tilde{V}_{1} \tilde{V}_{2}}{V_{1}^{(S)} V_{2}^{(S)}}=0.30$

\footnotetext{
${ }^{4}$ This particular value of $N$ is chosen using statistical reasoning, in order to guarantee that the semilength of the 99\% Agresti-Coull confidence interval will be less than 0.01 [51].
} 
Hence, according to the measure of overboundedness (15), the best model is the one generated via non-linear embedding and described by the matrices $A_{i}^{(4)}$. In general, models obtained via non-linear embedding tend to be less conservative than the ones obtained via sector non-linearity concept. This is probably due to the fact that the nonlinear embedding method tries to find the maximum and minimum value of $f(x) / x_{i}$, whereas the other method finds the maximum and minimum value of $\partial f(x) / \partial x_{i}$. Then, according to the mean-value theorem, $f(x) / x_{i}$ is bounded by $\partial f(x) / \partial x_{i}$, so that the extreme values of the former are smaller than those of the latter.

To conclude the comparison between the models, let us consider the measure based on the region of attraction as introduced in Section 3.2, with controllers designed applying Theorem 2 to the following LMI region:

$$
\mathcal{D}=\{z \in \mathbb{C}: \operatorname{Re}(z)<-1\}
$$

Figs. 3 and 4 show the phase-space (red arrows), the trajectories (blue lines) obtained starting from a grid of possible initial conditions (blue circles) and the region of attraction estimated using Theorem 1 (black dots) for the models generated via nonlinear embedding with matrices $A_{i}^{(3)}$ and the models generated via sector non-linearity concept (non-conservative). It can be seen that in all the cases, the trajectories converge towards the hyperellipsoid and eventually go to the origin.

The measure $M_{\beta}$ defined in (21) has been calculated for each model, giving the following results:

Model generated via non-linear embedding $A_{i}^{(3)}$ :

$$
M_{\beta}=\frac{V_{\beta}}{V_{\Theta}}=\frac{122.7323}{248.0502}=0.4948
$$

Model generated via non-linear embedding $A_{i}^{(4)}$ :

$$
M_{\beta}=\frac{V_{\beta}}{V_{\Theta}}=\frac{122.8700}{248.0502}=0.4953
$$

Model generated via sector non-linearity concept:

$$
M_{\beta}=\frac{V_{\beta}}{V_{\Theta}}=\frac{122.7605}{248.0502}=0.4949
$$

Model generated via sector non-linearity concept (conservative):

$$
M_{\beta}=\frac{V_{\beta}}{V_{\Theta}}=\frac{122.6771}{248.0502}=0.4946
$$

It can be seen that, also in this case, the model generated via non-linear embedding with matrices $A_{i}^{(4)}$ performs slightly better than the others, thus confirming to be the best obtained model. 


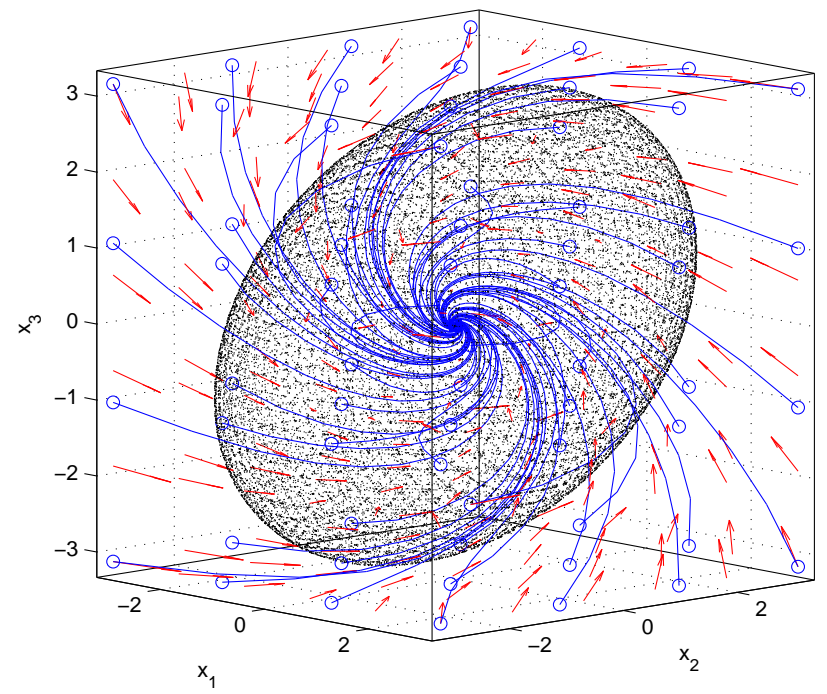

Figure 3: Phase-space, trajectories and estimated region of attraction for the closed-loop system obtained using the model generated via non-linear embedding with matrices $A_{i}^{(3)}$.

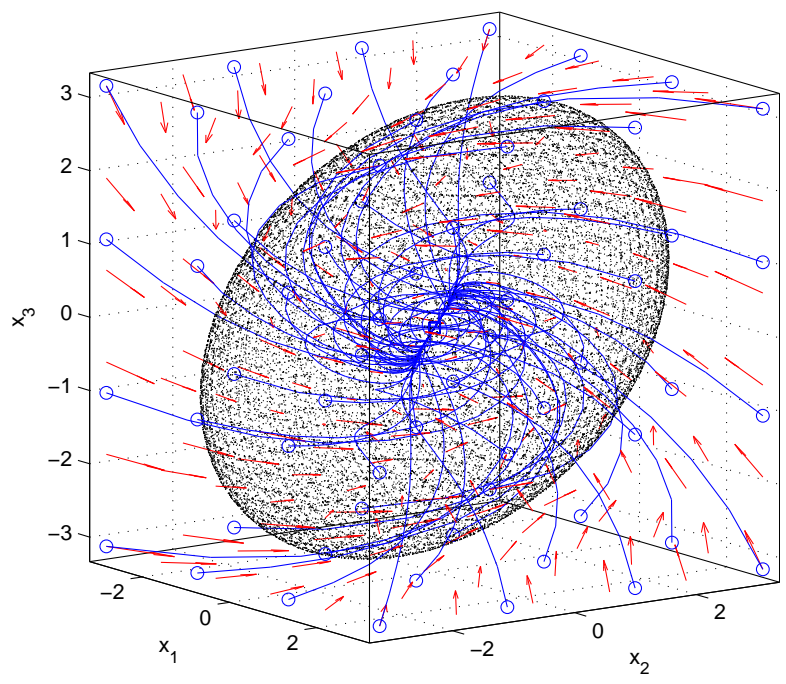

Figure 4: Phase-space, trajectories and estimated region of attraction for the closed-loop system obtained using the model generated via sector non-linearity concept. 


\section{Conclusions}

In this paper, the presence of strong analogies between polytopic LPV and TS systems and the automated generation of polytopic LPV and TS models have been addressed. In particular, it has been shown that the method for the automated generation of LPV models by non-linear embedding can be easily extended to generate automatically TS models from a given non-linear system. Analogously, a method already used in the TS framework for finding a model that describes in a fuzzy way a given non-linear function has been extended to the case of polytopic LPV description of non-linear systems.

Results obtained with a mathematical example have been presented and it has been shown, using an overboundedness measure, that the automatic generation via nonlinear embedding provides less conservative models than the automated generation via sector non-linearity concept. Also, a measure based on the region of attraction estimates has been introduced for comparing the closed-loop performances of the different models.

The overboundedness measure has shown to be an objective criterion that can be used to select which model can be considered the best. However, in the general case, which model is the best also depends on the context in which the model is used, i.e. whether it is used for stabilization or observation, and which structure of controller/observer is used for achieving the desired goal. Some information in this sense has been provided by the measure based on the region of attraction estimates, that allows comparing the closed-loop performances obtained with the different models when considering a quadratic $\mathcal{D}$-stabilizing state-feedback controller in the design step. The proposed measure could be easily extended to other controller structures, e.g. outputfeedback controllers, and to the observation case. However, it has the limit of providing an indication of which model is the best only a posteriori. It seems clear that an important path for future research is the development of a procedure that automatically selects the best model during the design step, taking into account what the model is used for and the used controller/observer structure.

\section{Acknowledgment}

This work has been funded by the Spanish Ministry of Science and Technology through the projects CICYT SHERECS (ref. DPI2011-26243) and CICYT ECOCIS (ref. DPI2013-48243-C2-1-R), by the European Commission through contract i-Sense FP7-ICT-2009-6-270428, by UPC through the grant FPI-UPC E-01104, by AGAUR through the contracts FI-DGR 2013 (ref. 2013FIB00218) and FI-DGR 2014 (ref. 2014FI_B1 00172), and by the DGR of Generalitat de Catalunya (SAC group Ref. 2014/SGR/374). The work was also supported by the National Science Centre in Poland under the grant 2013/11/B/ST7/01110.

\section{References}

[1] D. J. Leith, W. E. Leithead, Survey of gain-scheduling analysis design, International Journal of Control 73 (2000) 1001-1025. 
[2] J. S. Shamma, Analysis and design of gain scheduled control systems, Ph.D. thesis, Massachussets Institute of Technology, Department of Mechanical Engineering, advised by M. Athans, 1988 .

[3] J. S. Shamma, An overview of LPV systems, in: J. Mohammadpour, C. Scherer (Eds.), Control of linear parameter varying systems with applications, Springer, 2012, pp. 3-26.

[4] P. C. Pellanda, P. Apkarian, H. D. Tuan, Missile autopilot design via a multichannel LFT/LPV control method, International Journal of Robust and Nonlinear Control 12 (2002) 1-20.

[5] V. Verdult, M. Lovera, M. Verhaegen, Identification of linear parameter varying state space models with application to helicopter rotor dynamics, International Journal of Control 77 (2004) 1149-1159.

[6] D. Rotondo, F. Nejjari, V. Puig, Quasi-LPV modeling, identification and control of a twin rotor MIMO system, Control Engineering Practice 21 (2013) 829-846.

[7] G. J. Balas, Linear parameter-varying control and its application to a turbofan engine, International Journal of Robust and Nonlinear Control 12 (2002) 763796.

[8] F. D. Bianchi, R. J. Mantz, C. F. Christiansen, Gain scheduling control of variablespeed wind energy conversion systems using quasi-LPV models, Control Engineering Practice 13 (2005) 247-255.

[9] Z. Yu, H. Chen, P. Woo, Gain scheduled LPV $H_{\infty}$ control based on LMI approach for a robotic manipulator, Journal of Robotic Systems 19 (2002) 585-593.

[10] P. Gaspar, I. Szaszi, J. Bokor, Active suspension design using linear parameter varying control, International Journal of Vehicle Autonomous Systems 1 (2003) 206-221.

[11] B. Boulkroune, I. Djemili, A. Aitouche, V. Cocquempot, Robust nonlinear observer design for actuator fault detection in diesel engines, International Journal of Applied Mathematics and Computer Science 23 (2013) 557-569.

[12] S. Montes De Oca, V. Puig, M. Witczak, L. Dziekan, Fault-tolerant control strategy for actuator faults using lpv techniques: application to a two degree of freedom helicopter, International Journal of Applied Mathematics and Computer Science 22 (2012) 161-171.

[13] T. Takagi, M. Sugeno, Fuzzy identification of systems and its applications to modeling and control, IEEE Transactions on Systems, Man, and Cybernetics SMC-15 (1985) 116-132.

[14] X.-H. Yuan, J.-C. Ren, Y.-J. He, F.-C. Sun, Synthesis of $H_{2}$ guaranteed cost fuzzy controller for missile altitude system via linear matrix inequalities, in: Proceedings of the 2004 American Control Conference, volume 3, pp. 23092313. 
[15] Ł. Dziekan and M. Witczak and J. Korbicz, Active fault-tolerant control design for Takagi-Sugeno fuzzy systems, Bulletin of the Polish Academy of Sciences, Technical Sciences 59 (2011) 93-102.

[16] H.-S. Ko and K. Jatskevich, Power quality control of wind-hybrid power generation system using fuzzy-LQR controller, IEEE Transactions on Energy Conversion 22 (2007) 516-527.

[17] O. Begovich and E. N. Sanchez and M. Maldonado, Takagi-Sugeno fuzzy scheme for real-time trajectory tracking of an underactuated robot, IEEE Transactions on Control Systems Technology 10 (2002) 14-20.

[18] J. Cao and P. Li and H. Liu, An interval fuzzy controller for vehicle active suspension systems, IEEE Transactions on Intelligent Transportation Systems 11 (2010) $885-895$.

[19] D. Khiar and J. Lauber and T. Floquet and G. Colin and T. M. Guerra and Y. Chamaillard, Robust Takagi-Sugeno fuzzy control of a spark ignition engine, Control Engineering Practice 15 (2007) 1446-1456.

[20] D. Ichalal and B. Marx and J. Ragot and D. Maquin, New fault tolerant control strategies for nonlinear Takagi-Sugeno systems, International Journal of Applied Mathematics and Computer Science 22 (2012) 197-210.

[21] P. M. Mäkilä, P. Viljamaa, Convex parametric design, gain scheduling, and fuzzy computing, Technical Report, Tampere, Tampere University of Technology, Institute of Automation and Control, 2002.

[22] P. M. Mäkilä, P. Viljamaa, Linear programming based gain scheduling for LPV and PL systems, in: Proceedings of the 15th IFAC World Congress (2002).

[23] Q. Rong, G. W. Irwin, LMI-based control design for discrete polytopic LPV systems, in: Proceedings of the 6th European Control Conference (2003).

[24] P. Bergsten, R. Palm, D. Driankov, Observers for Takagi-Sugeno fuzzy systems, IEEE Transactions on Systems, Man, and Cybernetics - Part B: Cybernetics 32 (2002) 114-121.

[25] T. A. Johansen, R. Shorten, R. M. Smith, On the interpretation and identification of dynamic Takagi-Sugeno models, IEEE Transactions on Fuzzy Systems 8 (2000) 297-313.

[26] E. G. Collins, Book Review: Fuzzy control systems design and analysis: a linear matrix inequality approach, Automatica 39 (2003) 2011-2019.

[27] K. Tanaka, H. O. Wang, Fuzzy control systems design and analysis: A linear matrix inequality approach, John Wiley and Sons, Inc., 2001.

[28] K. Tanaka, H. Ohtake, H. O. Wang, Recursive pointwise design for nonlinear systems, in: Proceedings of the 2004 American Control Conference, volume 1, pp. $470-475$. 
[29] R. Toth, Modeling and identification of linear parameter-varying systems, Springer, 2010.

[30] A. Kwiatkowski, M.-T. Boll, H. Werner, Automated generation and assessment of affine LPV models, in: Proceedings of the 45th IEEE Conference on Decision and Control, San Diego, CA, USA (2006), pp. 6690-6695.

[31] H. Ohtake, K. Tanaka, H. O. Wang, Fuzzy modeling via sector nonlinearity concept, Integrated Computer-Aided Engineering 10 (2003) 333-341.

[32] P. Apkarian, P. Gahinet, G. Becker, Self-scheduled $H_{\infty}$ control of linear parameter-varying systems: A design example, Automatica 31 (1995) 1251 1261.

[33] J. S. Shamma, J. R. Cloutier, Gain-scheduled missile autopilot design using linear parameter varying transformations, Journal of Guidance, Control, and Dynamics 16 (1993) 256-263.

[34] M. Witczak, V. Puig, S. Montes De Oca, A fault-tolerant control strategy for nonlinear discrete-time systems: application to the twin-rotor system, International Journal of Control 86 (2013) 1788-1799.

[35] J. Bernoulli, Ars conjectandi, opus posthumum. Accedit Tractatus de seriebus infinitis, et epistola gallicé scripta de ludo pilae reticularis, Basel: Thurneysen Brothers, 1713.

[36] D. C. Montgomery, G. C. Runger, Applied statistics and probability for engineers, Wiley-Interscience, 1999.

[37] P. Apkarian, P. Gahinet, A convex characterization of gain-scheduled $H_{\infty}$ controllers, IEEE Transactions on Automatic Control 40 (1995) 853-864.

[38] P. Gahinet, P. Apkarian, M. Chilali, Affine parameter-dependent Lyapunov functions and real parametric uncertainty, IEEE Transactions on Automatic Control 41 (1996) 436-442.

[39] F. Bruzelius, S. Pettersson, C. Breitholtz, Region of attraction estimates for LPVgain scheduled control systems, in: Proceedings of the 7th European Control Conference.

[40] H. Khalil, Nonlinear systems, Prentice-Hall, Inc, Upper Saddle River, 1996.

[41] G. Becker, A. Packard, Robust performance of linear parametrically varying systems using parametrically-dependent linear feedback, Systems and Control Letters 23 (1994) 205-215.

[42] M. Chilali, P. Gahinet, $H_{\infty}$ design with pole placement constraints: An LMI approach, IEEE Transactions on Automatic Control 41 (1996) 358-367.

[43] B. A. Francis, A course in $H_{\infty}$ Control Theory, Lecture Notes in Control and Information Sciences, no. 88. Berlin: Springer-Verlag, 1987. 
[44] S. Gutman, E. I. Jury, A general theory for matrix root clustering in subregions of the complex plane, IEEE Transactions on Automatic Control AC-26 (1981) 853-863.

[45] A. S. Ghersin, R. S. Sanchez-Pena, LPV control of a 6 DOF vehicle, IEEE Transactions on Control Systems Technology 10 (2002) 883-887.

[46] D. Rotondo, F. Nejjari, V. Puig, A virtual actuator and sensor approach for fault tolerant control of LPV systems, Journal of Process Control 24 (2014) 203-222.

[47] T. M. Guerra, A. Kruszewski, J. Lauber, Discrete Tagaki-Sugeno models for control: Where are we?, Annual Reviews in control 33 (2009) 37-47.

[48] R. A. Horn, C. R. Johnson, Matrix analysis, Cambridge University Press, 1990.

[49] X.-D. Sun, I. Postlethwaite, Affine LPV modelling and its use in gain-scheduled helicopter control, in: UKACC International Conference on Control (1998), volume 2, pp. 1504-1509.

[50] S. Kawamoto, K. Tada, A. Ishigame, T. Taniguchi, An approach to stability analysis of second order fuzzy systems, in: Proceedings of the International Conference on Fuzzy Systems (1992), pp. 1427-1434.

[51] A. Agresti and B. A. Coull, Approximate is better than exact for interval estimation of binomial proportions, The American Statistician 52 (1998) 119-126. 